\title{
Flexibilidad curricular y titulación en la Universidad Tecnológica de Hermosillo, Sonora
}

\author{
María Guadalupe Marmolejo López
}

\section{Resumen}

Se exponen resultados parciales de la investigación sobre flexibilidad curricular y la culminación de estudios, a través de diversas formas de titulación. Los resultados son útiles a la Universidad Tecnológica de Hermosillo (UTH) donde existe una sola forma de titulación para egresar, la cual en algunos programas educativos no se adapta al sistema de evaluación de las competencias profesionales.

Para ello se observaron procesos de titulación para constatar el desarrollo metodológico en cuanto al nivel científico y académico del procedimiento; se estudió, a través de encuesta, el perfil del docente como: director, revisor o jurado, para evidenciar si cuenta con las habilidades metodológicas y de conocimiento para la implementación de las diferentes formas de titulación; se revisaron documentos, entre ellos el Reglamento de Titulación con la intención de realizar una propuesta de actualización.

Se aplicaron cuestionarios a estudiantes con el fin de determinar sus preferencias de titulación y motivación sobre la flexibilidad. Se encuestaron egresados para determinar la compatibilidad y efectividad de su titulación con el perfil de egreso y se entrevistaron empleadores para comprobar si las competencias adquiridas por los estudiantes aplican en el mercado laboral. Finalmente se proponen resultados que permiten trabajar sobre la flexibilidad educativa y las condiciones metodológicas para proponer diferentes formas de titulación.

\footnotetext{
* Doctorante en Educación Superior. Centro de Investigación y Educación Superior CIES-UNEPROP. Profesora de Tiempo Completo, Titular C. Universidad Tecnológica de Hermosillo, Sonora.
}

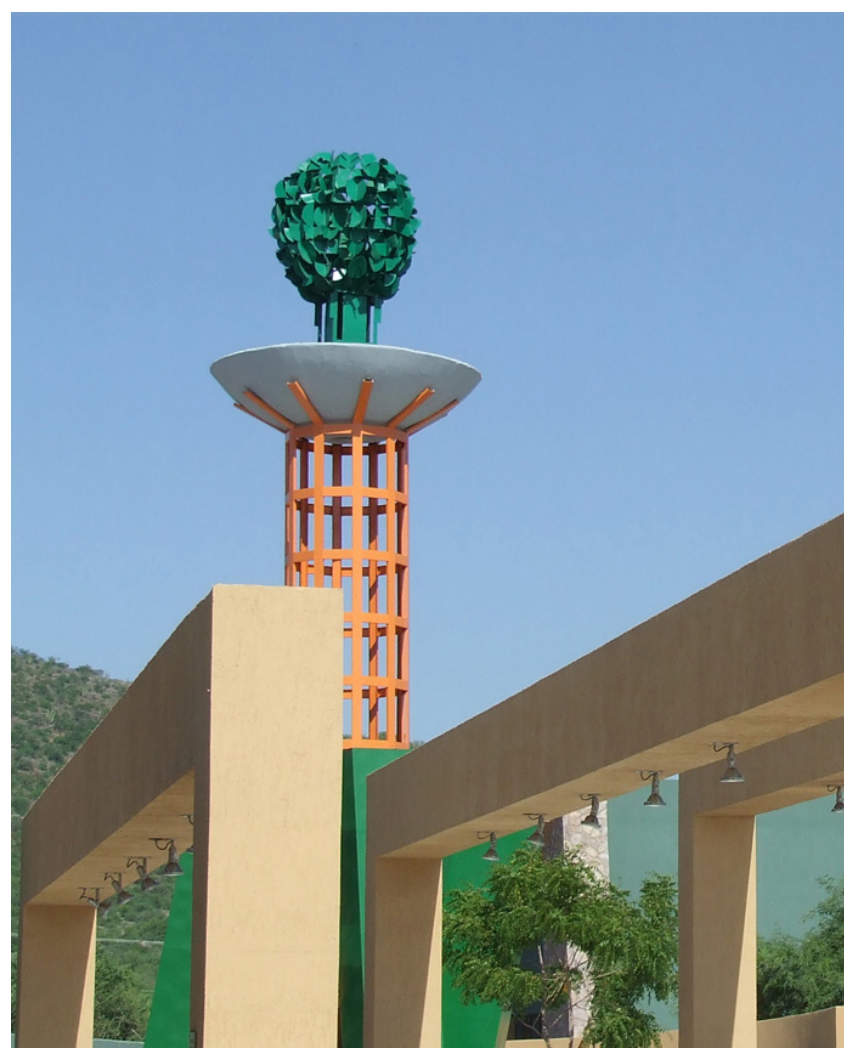

\section{Introducción}

El propósito de esta publicación se centra en un estudio que se traduce en postulados sobre la necesidad que tiene la Universidad Tecnológica de Hermosillo, Sonora de diseñar, para cada programa educativo, nuevas alternativas de titulación que fortalezcan y posibiliten a los futuros técnicos superiores universitarios trazar trayectoria flexible en su formación académica, 
que cumpla con sus expectativas de vocación y motivación por la carrera, tanto en su desarrollo futuro profesional como en su formación integral.

La flexibilidad curricular es un concepto diferente al de currículo flexible, ya que en este último solo se consideran materias opcionales del plan de estudio; sin embargo, la selección de trayectoria flexible del estudiante implica que los alumnos pueden contribuir a su formación desde su inicio hasta la culminación de su formación académica, escogiendo asignaturas alternativas que ofrece el currículo, su tema de titulación, su director de tesis y finalmente tomar la decisión; porque existen opciones múltiples, su acción de movilidad, de participación en congreso, su práctica profesional o social y su forma de titulación. Esto es a lo que nos referimos en esta investigación con trayectoria flexible de los estudiantes.

Para contribuir a lograr trayectorias flexibles de los estudiantes en los programas educativos de la UTH, se deben considerar diversas formas de titulación teniendo en consideración las características individuales de los programas educativos y las perspectivas futuras de los estudiantes.

En el Subsistema de Universidades Tecnológicas se promueve la flexibilidad de estructuras y planes de estudio para facilitar la formación multidisciplinaria, la integración del aprendizaje con la aplicación pertinente del conocimiento, así como el paso fluido de los estudiantes entre distintas instituciones. El modelo educativo opera con parámetros de alta calidad, monitoreados mediante periódicas evaluaciones internas y externas, lo cual asegura su constante evolución en términos de pertinencia respecto de los sectores sociales y laborales.
Para que esto pueda ocurrir, los programas educativos están diseñados con la flexibilidad suficiente para ser revisados y reorientados continuamente, de acuerdo con las necesidades manifestadas por los núcleos productivos, públicos y privados, de la zona de influencia de cada universidad tecnológica.

Las universidades tecnológicas surgieron en México en 1991 como organismos públicos descentralizados de los gobiernos estatales. El modelo educativo se sustenta en seis atributos que enmarcan y orientan el desarrollo curricular y su aplicación, como en la función de vinculación: calidad, pertinencia, intensidad, continuidad, polivalencia y flexibilidad.

La estructura curricular se sustenta en programas diseñados con base en competencias profesionales y está definida por tres niveles educativos: El primero corresponde al nivel de Técnico Superior Universitario (TSU), que se cursa en seis cuatrimestres de tiempo completo que son estudios intensivos, de 2625 horas presenciales y una estadía profesional de 525 horas; sus contenidos curriculares son $70 \%$ prácticos y $30 \%$ teóricos, forma profesionistas que se desempeñan en niveles de mando medio y responden a las demandas actuales del sector productivo.

El segundo corresponde al nivel Licenciatura o Ingeniería, que se cursa en cinco cuatrimestres adicionales, con duración de 1500 horas, más una estadía profesional de 480 horas; sus contenidos curriculares son $60 \%$ prácticos y $40 \%$ teóricos, son de carácter profesionalizante, forma mandos superiores y además proporcionan competencias relacionadas con la investigación aplicada y el desarrollo tecnológico. Es condición para que los alumnos puedan acceder al nivel de Licenciatura, invariablemente, el haber obtenido el

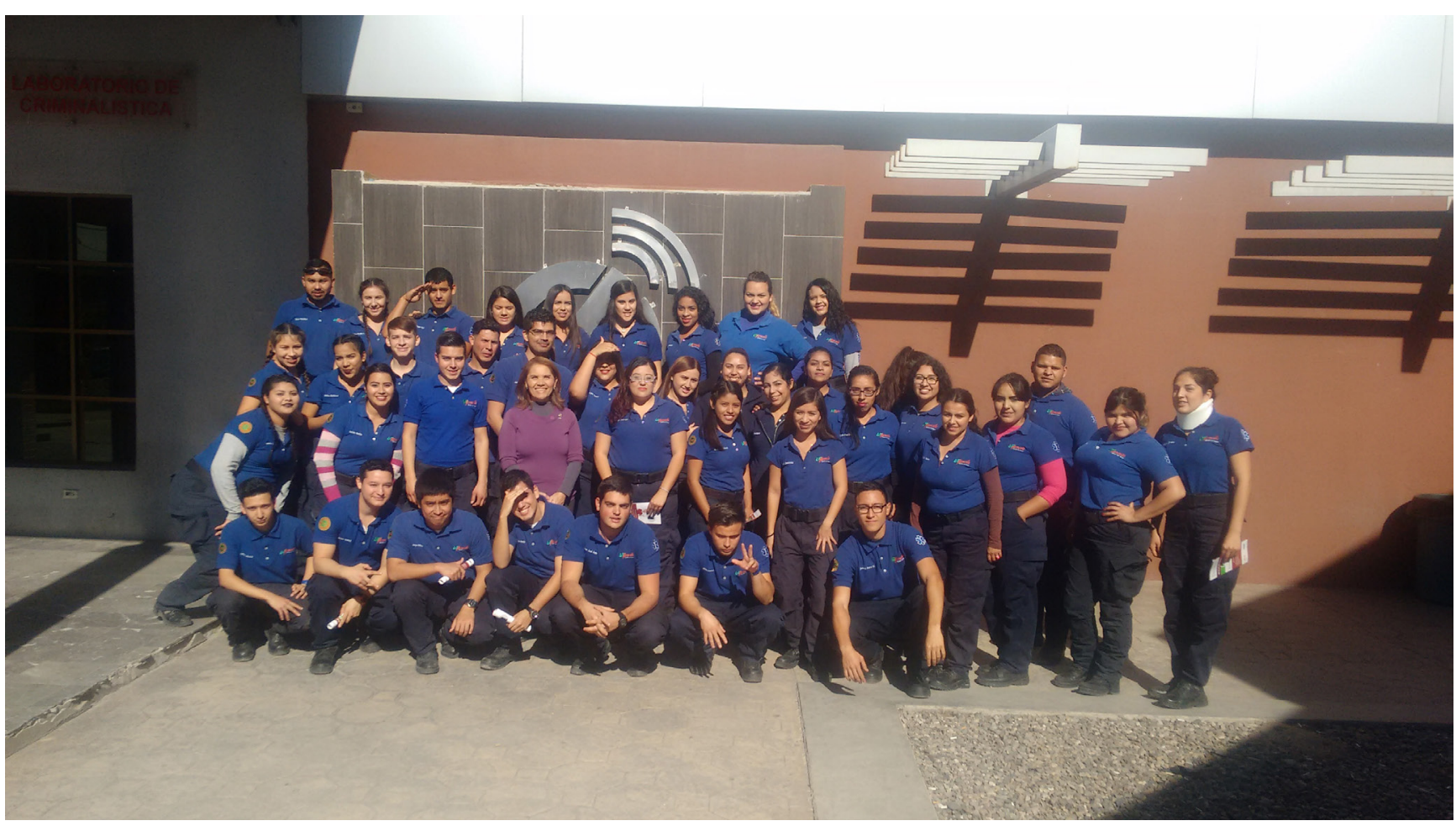




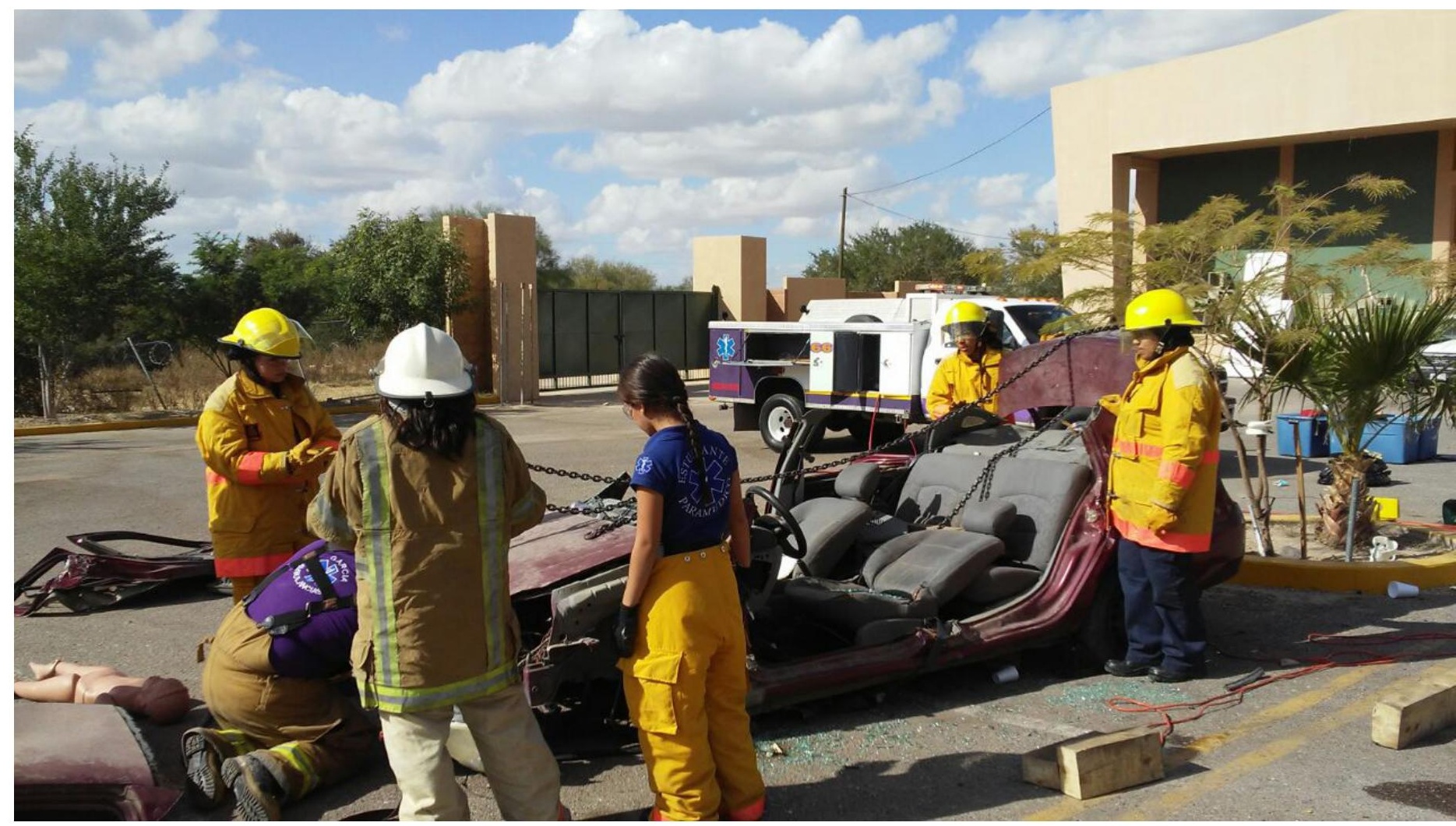

título profesional de TSU y el haber realizado y aprobado su estadía, la cual servirá además para acreditar su servicio social.

La tercera opción del modelo curricular permite que después de obtener el título de TSU, los estudiantes puedan optar por estudiar una ingeniería técnica, la cual se cursa en tres cuatrimestres con una duración de 800 horas, más una estadía de 480 horas; es de carácter altamente profesionalizante al preparar a los egresados en áreas específicas del ejercicio profesional para su inserción directa en el mercado laboral (Coordinación General de Universidades Tecnológicas y Politécnicas (UTyP), Subsecretaría de Educación Superior, 2013).

De acuerdo con Escalona Ríos (2008), en términos generales, la flexibilidad curricular se refiere a la movilidad interna y externa de un currículo, es decir, de un plan de estudios. Esta flexibilidad se da con la interdisciplinariedad, la integración, las competencias profesionales y los sistemas de créditos, entre otras cosas. Así, la flexibilidad plantea una diversidad de opciones de educación que pretende alcanzar un nivel equitativo de competencias educativas.

La flexibilidad es un elemento básico que coadyuva a la sociedad del conocimiento para romper las estructuras de tiempo y espacio en aras de adquirir un conocimiento que transforme las relaciones sociales de aprendizaje, a partir del redimensionamiento de las prácticas pedagógicas, ya que no es necesario compartir un espacio físico para compartir un conocimiento ni tampoco tener una relación sincrónica.
De esta forma, la flexibilidad curricular implica abrir los espacios rígidos, coordinados por normas y reglas institucionales, para darle paso a una formación profesional en la que el actor principal es el alumno y sus intereses profesionales. Cabe mencionar que, según Romero, citado por Escalona Ríos (2008), la única finalidad de esto es abrir los procesos de enseñanza-aprendizaje cuyas características son:

a) Perseguir permanentemente los nuevos conocimientos que plantean los procesos de formación

b) Promover la capacidad de decisión en el alumno para que pueda elegir los créditos que debe cursar

c) Propiciar la comunicación horizontal y vertical de los contenidos

d) Optar por el aprendizaje integral mediante el contacto con el mundo circundante

e) Vincular a la universidad con distintas instituciones de la sociedad

f) Estimular la interdisciplinariedad.

La flexibilidad implica buscar una mayor articulación o integración de las unidades y agentes responsables de la dirección y la gestión de todos estos procesos. En este sentido, exige también acciones de concertación política y académica alrededor de compromisos establecidos, a la vez de tareas de innovación y cambio de propuestas. La noción de flexibilidad curricular o de currículo flexible se ha ido convirtien- 


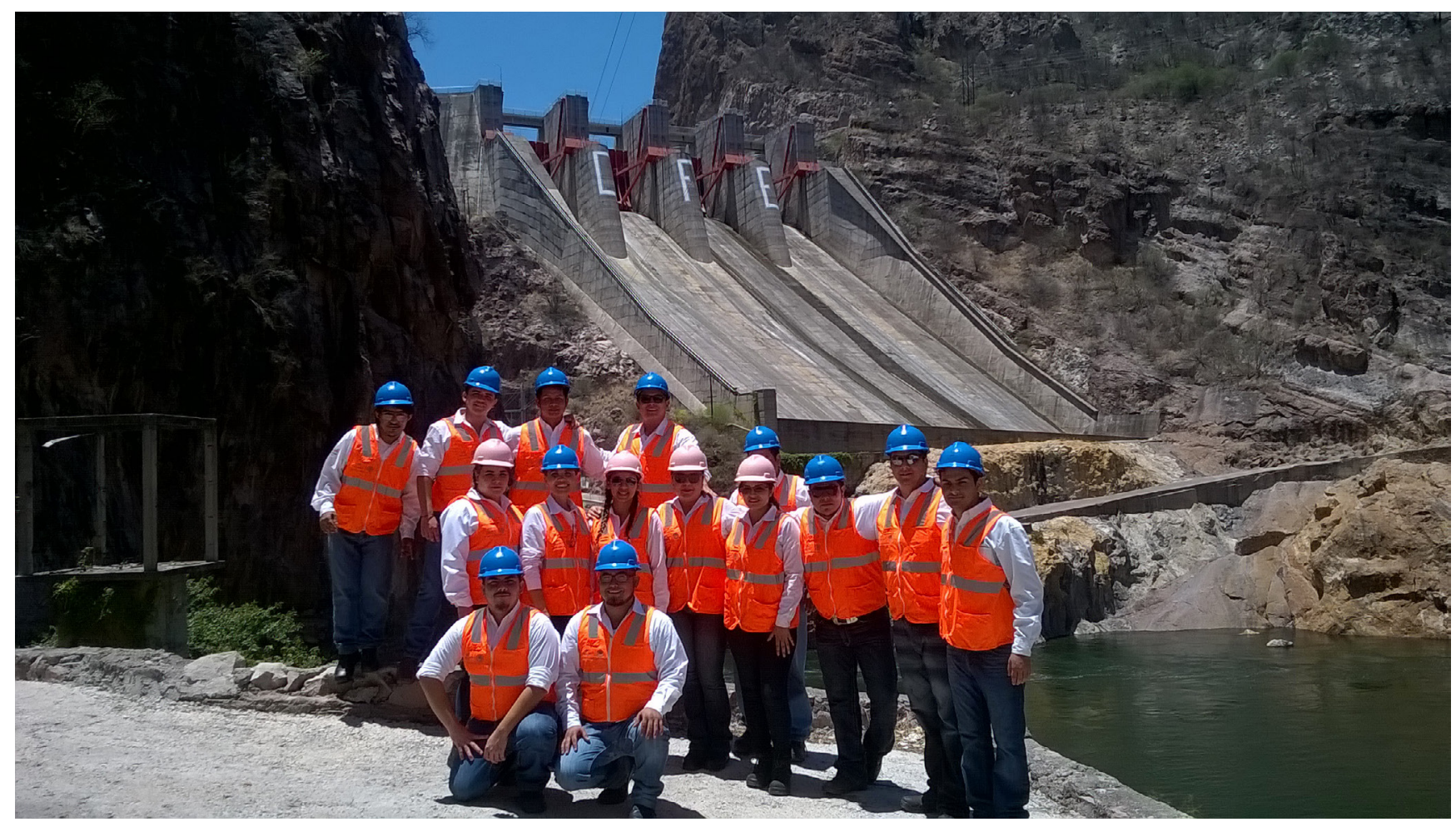

do en una herramienta metodológica que le otorga un nuevo sentido a los diferentes aspectos que plantean las reformas académicas en la educación superior.

Desde este punto de vista, la noción de flexibilidad curricular se asocia, como marco general, a reformas de la educación superior en aspectos tales como la reorganización académica, el rediseño de programas académicos y de sus planes de estudio, la transformación de los modelos de formación tradicional, la redefinición del tiempo de formación, una mayor asociación de la formación con las demandas del entorno laboral, etcétera.

Al abordar la flexibilidad curricular en su dimensión estructural, se plantean dos tipos de estrategias:

Unas centradas en modificar los patrones organizativos del conocimiento; esto es, en abrir los límites entre campos, áreas y unidades de conocimiento que conforman el currículo, de modelos curriculares organizados con base en disciplinas a modelos curriculares más integrados.

Otras que buscan la ampliación y diversificación de la oferta educativa para satisfacer mejor las demandas sociales y los intereses del estudiante, brindándoles mayores grados de autonomía en la organización de su programa de estudios.

En ambas modalidades, la flexibilidad implica una reconceptualización de las formas tradicionales y una mayor articulación de los conocimientos que se producen y trasmiten, con los intereses estudiantiles y con las demandas del entorno social (Escalona Ríos, 2008).
En la medida que la sociedad, los medios de comunicación y la tecnología, entre otros, evolucionan, es obvio que el currículo requiere de actualización. En este contexto, Rocío Soto (1993), en su artículo Propuesta para un Modelo Curricular Flexible, señala que los sistemas educativos se encuentran en correspondencia directa con los modelos de desarrollo de una realidad social dada.

Los modelos actuales tienden hacia la apertura comercial y la internacionalización de la economía, características que les confieren propiedades diferentes a la demanda educativa. Enfrentar los nuevos retos que representan los modelos actuales de desarrollo implica, en el caso de la educación superior, asumir el compromiso y la responsabilidad que les confiere el desarrollo de la tecnología, la investigación y la producción de conocimientos, dando mayor versatilidad a sus tradicionales funciones sustantivas de docencia, investigación y extensión.

En consecuencia, existe una gran demanda social para que la educación superior mejore la calidad académica de sus servicios, produzca mejores egresados, se modernice y retome el liderazgo en las áreas estratégicas para el desarrollo nacional (Mendoza Rojas, 1993), de tal manera que realice su labor educativa, dando mayor importancia a los objetivos de calidad, interdisciplinariedad, vinculación y responsabilidad social en la realización de sus funciones sustantivas.

En virtud de ello, se intensifican algunas líneas de acción y se corrigen otras, impulsando estrategias de corresponsabilidad hacia la madurez y capacidad para la labor de conjunto, en la planeación, ejecución y eva- 
luación de programas de superación académica que permitan atender con mejor calidad la demanda educativa, diversificar la formación del estudiante para hacerlo un profesional más flexible; todo esto, como una actitud emprendedora hacia el trabajo y la producción, fomentando el autoaprendizaje, el ejercicio interdisciplinario y la actualización permanente.

Refiere además que el currículo flexible, en su forma operativa, se define como una propuesta alternativa a la concepción lineal y rígida de los estudios profesionales, que rompe con el sistema de materias y cursos seriados y obligatorios, y presenta en su lugar una amplia gama de opciones para la formación profesional del estudiante. Se vincula directamente al desarrollo histórico de su realidad -con un carácter prospectivo- adaptable a las continuas modificaciones de su contexto y posibilitador del flujo de docentes, investigadores, estudiantes, experiencias y actividades; se convierte en un proyecto educativo que conceptualiza y orienta tanto el proceso institucional como el profesional y el individual (Soto Perdomo, 1993).

La carrera de TSU Paramédico se crea en la UTH en el año 2002. En el 2012 se extendió el plan de estudios para transitar a la Licenciatura en Protección Civil y Emergencias. No obstante, este notable avance en el modelo académico, permaneció igual en el esquema de titulación.

Una de las metas de la educación superior actual, es elevar el papel protagónico en el proceso educativo de los estudiantes, a través de actividades de movilidad, vinculación y flexibilización de la educación universitaria fundamentada en diseños curriculares flexibles y elección de los estudiantes de trayectorias flexibles de su formación. Lo que significa que los estudiantes desde su inicio en un sistema educativo pueden elegir las asignaturas opcionales que complementan su formación, además de poder elegir la temática de tesis, su director, la participación en congresos o eventos locales, nacionales e internacionales, así como su acción de movilidad y hasta inclusive su forma de titulación según sus intereses de posgraduados.

Hablar de un currículo flexible para el área de la salud, no se circunscribe al hecho de relacionarlo con la actividad social, sino en propiciar la interrelación de todas las asignaturas del plan. El paramédico, al igual que el médico, la enfermera y demás recursos humanos en esta esfera, deben aprender y formarse en función de una educación interdisciplinaria.

En el plan de estudios de la carrera de TSU Paramédico de la Universidad Tecnológica de Hermosillo, Sonora, se identifican cuatro niveles del saber:

Nivel básico: conjunto de saberes esenciales para el aprendizaje de nuevos contenidos y que son comunes a todas las carreras de TSU; en este nivel se clasifican todas las materias de lenguajes y métodos, así como las de ciencias básicas que cumplan con este criterio.

Nivel genérico: los saberes comunes de la disciplina a la que pertenece la carrera; en este nivel se clasificarán todas las demás materias de ciencias básicas y aquellos conocimientos técnicos que cumplan con el criterio.

Nivel específico: saberes que diferencian a la carrera de otras de la misma disciplina; en este nivel se clasificarán aquellas materias de conocimientos técnicos que cumplan con este criterio.

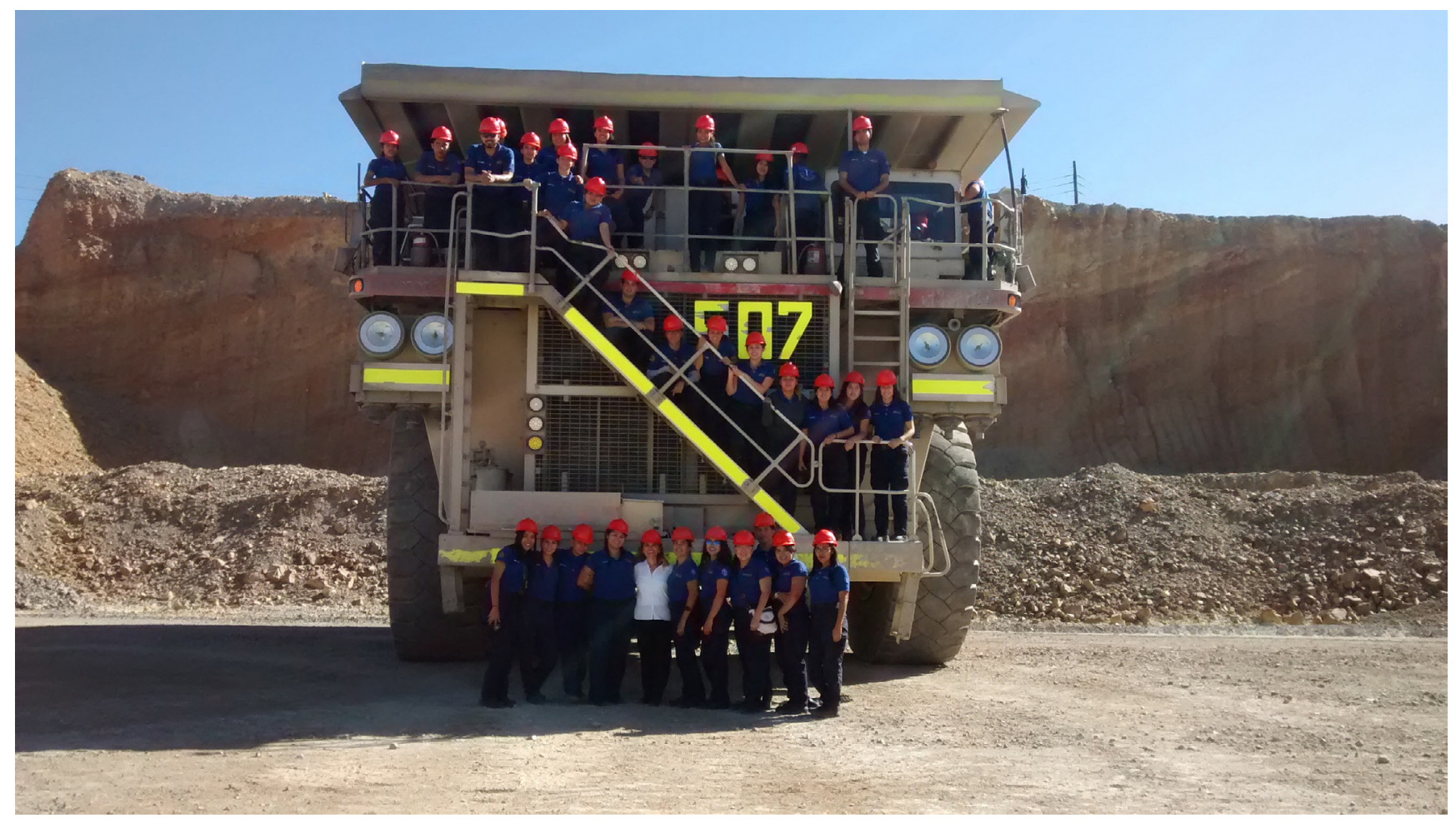




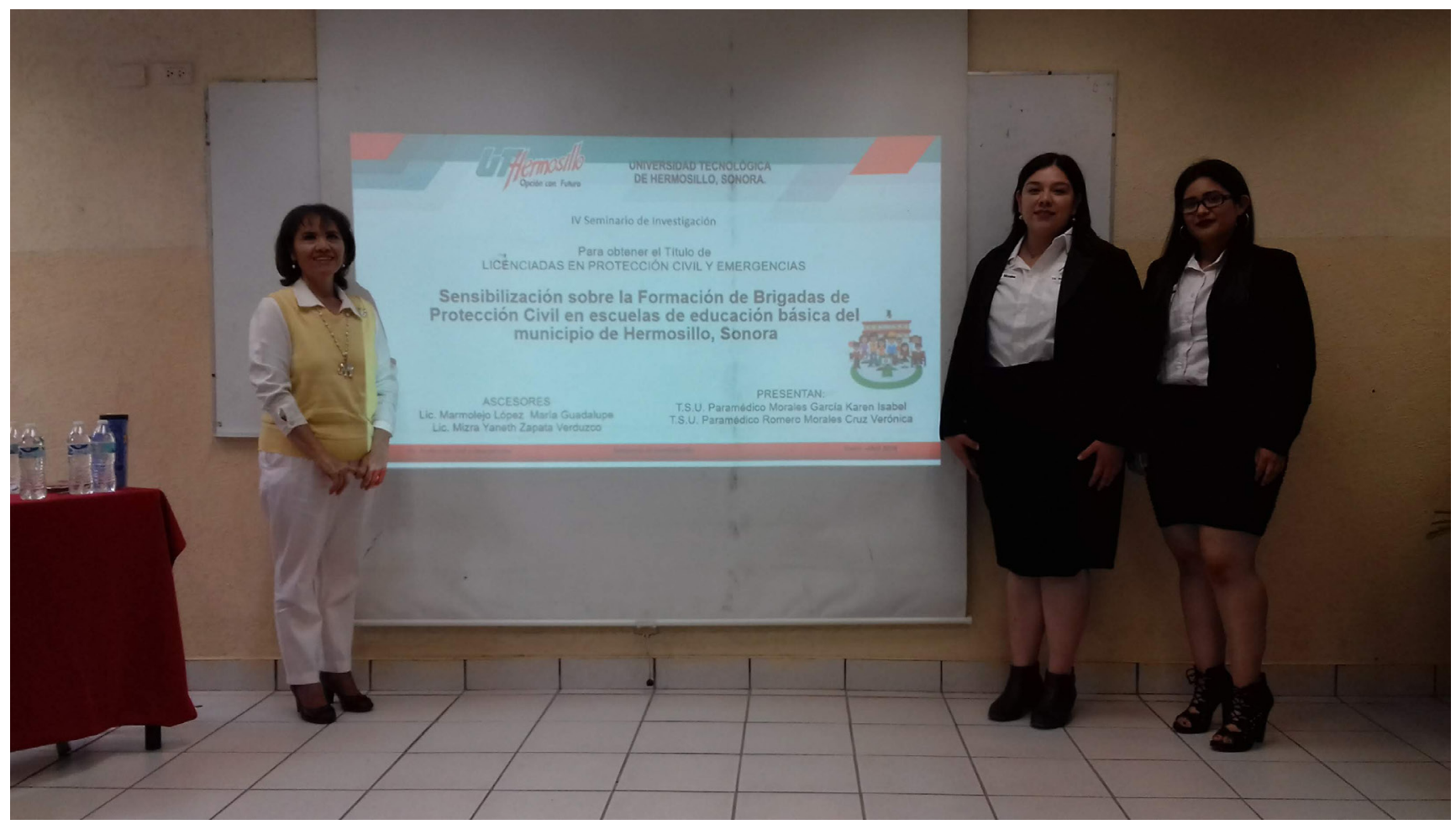

Nivel flexible: el subconjunto del plan constituido por aquellas materias del área de conocimientos técnicos de nivel específico que no son determinantes para la esencia del perfil profesional de la carrera particular, pero que responden a las demandas del entorno particular de la universidad. También lo conforman el conjunto de saberes referenciales del entorno sociocultural, integradas por las asignaturas del área de formación sociocultural.

El problema que se aborda en este trabajo de investigación se fundamenta en la necesidad de flexibilizar el currículo de formación de los alumnos, para que ellos puedan elegir la opción que más les parezca, entre las distintas formas de titulación; esto incide en la modificación del Reglamento de Titulación para anexar otras opciones; incrementar el número de horas para la asignatura de Metodología de la Investigación, así como colocarla en los primeros cuatrimestres de enseñanza, mediante el rediseño de las materias optativas; capacitar y actualizar al personal docente que funge como revisor de las memorias técnicas e implementar un modelo de titulación que favorezca la adquisición del perfil de egreso.

Las conclusiones de esta investigación se derivan de la observación de procesos de titulación para constatar el desarrollo metodológico en cuanto al nivel científico y académico del procedimiento; asimismo, del estudio, a través de encuestas, del perfil del docente como: director, revisor o jurado, que evidencia las habilidades metodológicas y de conocimiento para la implementación de las diferentes formas de titula- ción; también de la revisión del Reglamento de Titulación con la intención de realizar una propuesta de actualización; de la aplicación de cuestionarios a estudiantes con el fin de determinar sus preferencias de titulación y motivación sobre la flexibilidad, así como de las encuestas documentadas por los egresados para determinar la compatibilidad y efectividad de su titulación con el perfil de egreso; y de las entrevistas a los empleadores para comprobar si las competencias adquiridas por los estudiantes aplican en el mercado laboral.

Los datos recopilados a través de los instrumentos fueron procesados en tablas dinámicas de Excel, sobresaliendo lo siguiente:

El crecimiento y la dinámica en la que se halla inmersa la UTH por la evolución que ha tenido, al transitar de formar técnicos superiores universitarios a egresar licenciados e ingenieros; es decir, incrementar el grado académico de nivel 5B a nivel 5A (CINE), ${ }^{3}$ exige flexibilizar el currículo con nuevas alternativas de titulación que fortalezcan y posibiliten a los futuros técnicos superiores universitarios trazar trayectoria flexible para su formación académica, que cumpla con sus expectativas de vocación y motivación por la carrera, tanto en su desarrollo futuro profesional como en su formación integral.

Los estudiantes requieren fortalecer la competencia redactora (comunicación escrita); para ello, se propone modificar los contenidos de las materias optativas introduciendo la temática de Metodología de la Investigación desde los primeros cuatrimestres. 
El Reglamento de Titulación debe actualizarse para insertar nuevas opciones de titulación que amplíen las posibilidades de los estudiantes.

El $20 \%$ de los egresados se encuentra laborando en áreas que no están relacionadas con su perfil de egreso debido a que no se sienten seguros para ejercer profesionalmente como paramédico.

Los empleadores demandan la aplicación de instrumentos que permitan comprobar que el estudiante ha logrado las competencias, habilidades y destrezas, al margen de que señalan como torales las actitudes que enmarca el perfil de egreso.

\section{Metodología}

En la investigación realizada se escogieron la población y la muestra de la carrera de TSU Paramédico; la cual, por su perfil profesional, permite en su currículo la aplicación de diferentes tipos de opciones de titulación, y por la similitud en la forma actual de titulación de los estudiantes se comporta como una muestra representativa de cualquiera de los sistemas educativos de la UTH. Los datos recopilados, a través de los instrumentos asociado a los métodos de encuesta y test, fueron procesados en tablas dinámicas de Excel.

En la investigación, algunos de los resultados propuestos fueron obtenidos con el conocimiento empírico adquirido a través de la observación de muchos años de procesos de titulación en algunos programas educativos de la UTH y de otras instituciones de enseñanza superior; en esos resultados, se prestó gran interés a la metodología y dinámica empleada para el desarrollo de la actividad, tanto desde el punto de vista del trabajo del estudiante como del jurado evaluador y de la participación del público.

El resultado de esta observación arrojó que en general, el proceso de titulación era siempre igual y de forma tradicional; en ese proceso, el estudiante defiende un tema en un tiempo promedio de quince a veinte minutos; después, el jurado hace preguntas en un tiempo promedio de quince minutos, con unos diez minutos de discusión para culminar el proceso. Otra de las deficiencias observadas es que las actividades de titulación tenían un alto grado de improvisaciones y no se escogían los mejores académicos como sinodales o jurados.

El estudiante presentaba poco interés por los temas de la monografía; además, no estaban vinculados con el modelo de actuación del profesional según el caso; también, la participación del público no era promovida por los dirigentes de los jurados, cuestión que le restaba dinámica, interés y motivación a la ceremonia de titulación.

Como parte de la elección de los métodos aplicables, se revisaron documentos en busca de un estatuto de titulación con indicaciones metodológicas precisas sobre todo en el proceso de titulación con la reglamentación, desde su inicio con la selección del tema, hasta el final con el acto o ceremonia de titulación. En la revisión encontramos que solo existe una forma de titulación en el programa educativo de TSU Paramédico, y no existen indicaciones metodológicas precisas y adecuadas, organizativas y administrativas que regulen la titulación.

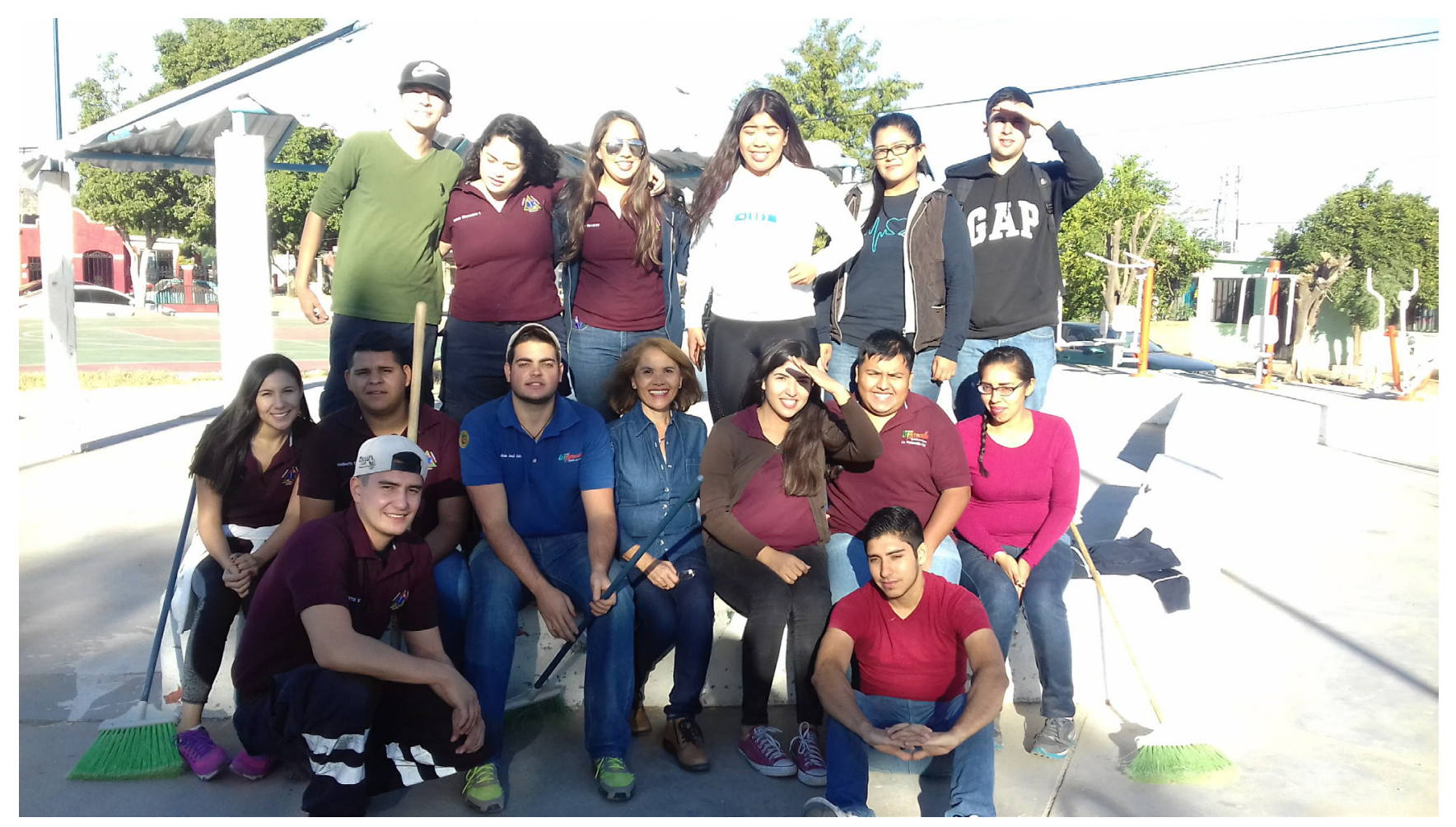


Por otro lado, se aplicó una encuesta a los maestros del programa educativo de TSU Paramédico, continuidad a la Licenciatura en Protección Civil y Emergencias, con el objetivo de medir el estado de conocimiento y su motivación para participar en las actividades de titulación. La encuesta presentaba quince reactivos y se aplicó al $90 \%$ de la planta académica. El $75 \%$ de la misma mostraron un desinterés para participar en las actividades de titulación, mostrando poco conocimiento sobre la importancia de la actividad y su relación con el modelo de actuación del profesional y el perfil de ingreso. El 50 \% presentaba desconocimiento total sobre el propósito y objetivo de la titulación o solo tenían conocimientos parciales de una sola forma de titulación y desconocimiento casi total sobre la flexibilidad y su relación con otras formas de titulación.

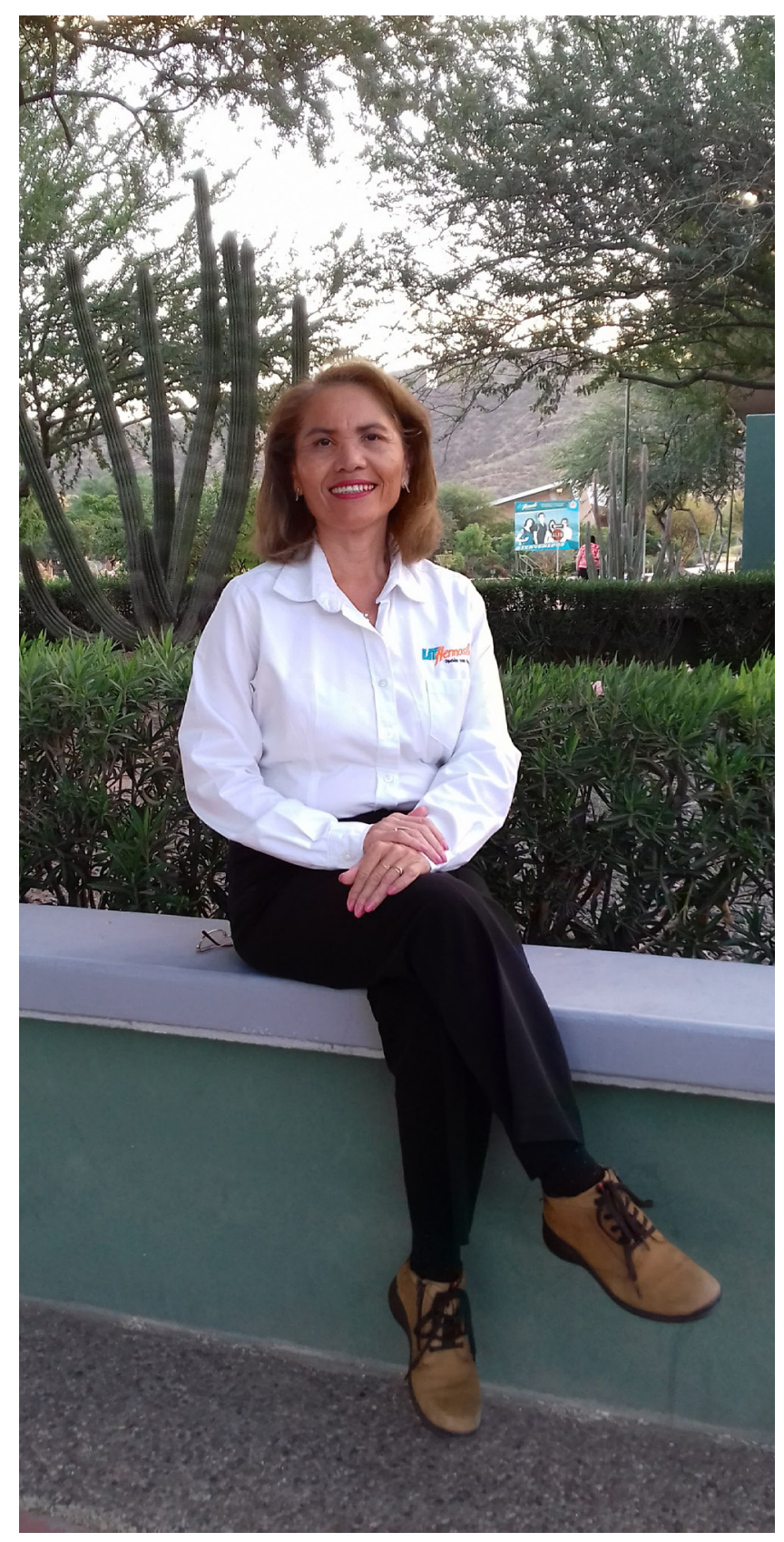

La encuesta a los alumnos presentaba el objetivo de medir su motivación sobre su participación en la organización de su formación con interés en la posibilidad de tener diferentes opciones de titulación. La muestra fue escogida de la carrera de TSU Paramédico, continuidad a la Licenciatura en Protección Civil y Emergencias. La tendencia de los alumnos es tener más participación en su formación, pero el $80 \%$ planteó que no tenían información de muchos aspectos de la carrera como: planes y programas de estudios, reglamento de estudiante, el perfil de egreso, cómo se pueden titular según sus intereses vocacionales. El $100 \%$ de los alumnos rechazan los currículos no flexibles y solicitaban información sobre el significado de trayectoria flexible.

Se entrevistaron a los profesores de tiempo completo de la carrera de TSU Paramédico con el objetivo de conocer cómo se organiza el proceso de titulación y qué actividades académicas se hacen con la planta docente para elevar el nivel de conocimientos acerca de la flexibilidad y la titulación y cuáles son las indicaciones metodológicas existentes para la organización del proceso de titulación desde su inicio hasta el final. Se detectaron muchas deficiencias, pues no existe un reglamento de trabajo de las academias y no se planifican reuniones metodológicas para seleccionar a los académicos que van a ser directores de tesis para la titulación; no existe un banco de temáticas o de problemas de tesis y tampoco se cuenta con un aparato de divulgación para que los alumnos tengan información.

Se aplicaron test y prueba a una muestra de los graduados de las generaciones que salieron tanto de la carrera de TSU Paramédico como de la Licenciatura en Protección Civil y Emergencias, con el fin de determinar la influencia de la forma de titulación tradicional con la continuidad de estudio entre TSU y la licenciatura, así como de los beneficios que han alcanzado en el ejercicio de la profesión; y ambos llegaron a la conclusión que la titulación actual no le aportó nada o muy poco en el desempeño profesional. Por otro lado, se entrevistaron a cinco empleadores, incluyendo a la dirección de la licenciatura, con el objetivo de conocer desde su punto de vista, sus preferencias para un mejor desempeño del estudiante sobre su forma de titulación.

\section{Resultados y discusión}

Los resultados que se exponen de este estudio representan la concreción del análisis de los datos recabados mediante encuestas, entrevistas y test aplicados a los sujetos participantes, así como de la observación sobre la evolución que ha tenido la carrera en cuanto a la retención de estudiantes y al aprovechamiento escolar.

El objetivo de este ejercicio científico fue exponer los resultados de la investigación realizada dentro de la carrera de TSU Paramédico, continuidad a la Licen- 
ciatura en Protección Civil y Emergencias, para demostrar que, tanto docentes como estudiantes, egresados y empleadores, expresaron afinidad y apertura con respecto a la flexibilización del plan de estudios, que les permita a los alumnos elegir, desde un inicio, su trayectoria académica, las materias opcionales, el tema de titulación, así como al asesor institucional que les acompañará en el desarrollo de su proyecto de estadías.

Por su parte, los docentes se mostraron sensibles a este cambio asumiendo las ventajas que les representa el contar con un Reglamento de Titulación en el que se establezcan las indicaciones metodológicas para este proceso de principio a fin.

En cuanto a la aplicación de los métodos teóricos, al resultado de los métodos empíricos aplicados y las deficiencias detectadas se propone hacer una reunión metodológica de carrera de TSU Paramédico, continuidad a la Licenciatura en Protección Civil y Emergencias, para incorporar a la forma actual de titulación otras formas diferentes para el TSU y la Licenciatura, que considere para el nivel $5 \mathrm{~B}$ la titulación por promedio, si va a continuar con nivel $5 \mathrm{~A}$; además, la titulación por examen general de conocimiento y por práctica profesional, siendo la titulación por tesis selectiva para estimular a los mejores estudiantes.

Se recomienda crear una comisión académica colegiada para elaborar el Reglamento de Titulación donde exista la reglamentación administrativa, metodológica y de organización docente de todas las formas de titulación. Que se hagan reuniones de academias para dar las indicaciones metodológicas de las diferentes formas del proceso de titulación y seleccionar bancos de asesores y temas. Que se divulgue a través de la página de internet de la carrera para que los estudiantes puedan escoger, tanto la forma de titulación, temas y asesores para participar activamente en la flexibilización de su formación. Hacer talleres o festival estudiantil para dar a conocer el objetivo de cada forma de titulación y sus ambiciones profesionales para elevar la dinámica y motivación por la carrera.

El currículo es el punto de partida del proceso de enseñanza-aprendizaje, y la tendencia actual es direccionarlos hacia el carácter activo del estudiante, a la personalización del proceso, la creatividad, la comunicación entre ellos, el vínculo escuela-sociedad, el papel esencial del maestro y el aprendizaje significativo, entre otros; por lo tanto, una de las características más substanciales que debe tomarse en cuenta dentro de la metodología para la conformación de un programa de estudios aplicable, en estos tiempos de modernidad, que sobresale en todos los ámbitos, es la flexibilidad.

\section{Conclusiones}

El grado académico en el Subsistema de Universidades Tecnológicas y Politécnicas se amplió en el año
2009, sin embargo, se mantiene el mismo esquema de titulación desde 1991. La única opción de titulación vigente, "Memoria Técnica", resultado de las estadías en el sector productivo (15 semanas), no demuestra que los egresados hayan alcanzado el perfil de egreso.

Se requiere flexibilizar el currículo para incorporar nuevas modalidades de titulación que permitan al estudiante elegir su trazabilidad académica. De igual forma, es imperante modificar el Reglamento de Titulación para incluir los aspectos metodológicos suficientes para optimizar este proceso.

Hace falta fortalecer el perfil académico de los docentes que fungen como asesores institucionales.

Existe una gran demanda social para que la educación superior mejore la calidad académica de sus servicios, produzca mejores egresados, se modernice y retome el liderazgo en las áreas estratégicas para el desarrollo social.

La Universidad Tecnológica de Hermosillo, Sonora debe diseñar para cada programa educativo, nuevas alternativas de titulación que fortalezcan y posibiliten a los futuros técnicos superiores universitarios, trazar una trayectoria flexible para su formación académica, que cumpla con sus expectativas de vocación y motivación por la carrera, tanto en su desarrollo futuro profesional, como en su formación integral.

\section{Referencias}

Coordinación General de Universidades Tecnológicas y Politécnicas (UTyP), Subsecretaría de Educación Superior. (2013). Coordinación Académica: Modelo Educativo. Recuperado el 20 de febrero de 2018, de http://cqutyp.sep.gob.mx/Areas/CoordAcademica/index.php

Escalona Ríos, L. (2008). Flexibilidad curricular: elemento clave para mejorar la educación bibliotecológica. Investigación bibliotecológica, 22(44), 143-160. Recuperado el 25 de junio de 2018, de http://files.sld.cu/bmn/files/2014/07/super-flexibilidad curricular.pdf

Instituto de Estadística de la Organización de las Naciones Unidas para la Educación, la Ciencia y la Cultura (UNESCO). (2013). Clasificación Internacional Normalizada de la Educación CINE 2011. Recuperado el 30 de abril de 2018, de http://unesdoc.unesco.org/images/0022/002207/220782s.pdi

Mendoza Rojas, J. (1993). El proyecto de modernización universitaria: continuidades e innovaciones (pp. 1-18). Recuperado de http://publicaciones. anuies.mx/pdfs/revista/Revista84 S1A1ES.pdf

Soto Perdomo, R. (marzo, 1993). Propuesta para un Modelo Curricular Flexible. Revista de la Educación Superior, 85,1-9. Recuperado el 13 de mar$z 0$ de 2018, de htto://publicaciones.anuies.mx revista/85/2/4/es/propuesta-para-un-modelo-curricular-flexible 